\title{
MPICH-G2: A Grid-Enabled Implementation of the Message Passing Interface
}

\author{
Nicholas T. Karonis \\ Department of Computer Science \\ Northern Illinois University \\ DeKalb, IL 60115 \\ Argonne National Laboratory \\ Argonne, IL 60439 \\ Email:karonis@niu.edu \\ and \\ Brian Toonen \\ Mathematics and Computer Science Division \\ Argonne National Laboratory \\ Argonne, IL 60439 \\ Email: toonen@mcs.anl.gov \\ and \\ Ian Foster \\ Argonne National Laboratory \\ Argonne, IL 60439 \\ The University of Chicago \\ Chicago, IL 60637 \\ Email: foster@mcs.anl.gov
}

Version: April 2002

Proposed running head: MPICH-G2: A Grid-Enabled MPI 
Application development for distributed computing "Grids" can benefit from tools that variously hide or enable application-level management of critical aspects of the heterogeneous environment. As part of an investigation of these issues, we have developed MPICH-G2, a Grid-enabled implementation of the Message Passing Interface (MPI) that allows a user to run MPI programs across multiple computers, at the same or different sites, using the same commands that would be used on a parallel computer. This library extends the Argonne MPICH implementation of MPI to use services provided by the Globus Toolkit for authentication, authorization, resource allocation, executable staging, and I/O, as well as for process creation, monitoring, and control. Various performancecritical operations, including startup and collective operations, are configured to exploit network topology information. The library also exploits MPI constructs for performance management; for example, the MPI communicator construct is used for application-level discovery of, and adaptation to, both network topology and network quality-of-service mechanisms. We describe the MPICH-G2 design and implementation, present performance results, and review application experiences, including record-setting distributed simulations.

Key Words: MPI, Grid computing, message passing, Globus Toolkit, MPICH-G2 


\section{INTRODUCTION}

So-called computational Grids [18, 14] enable the coupling and coordinated use of geographically distributed resources for such purposes as large-scale computation, distributed data analysis, and remote visualization. The development or adaptation of applications for Grid environments is made challenging, however, by the often heterogeneous nature of the resources involved and the facts that that these resources typically live in different administrative domains, run different software, are subject to different access control policies, and may be connected by networks with widely varying performance characteristics.

Such concerns have motivated various explorations of specialized, often highlevel, distributed programming models for Grid environments, including various forms of object systems [26, 24], Web technologies [22, 50], problem solving environments [7, 45], CORBA, workflow systems, high-throughput computing systems [1], 39], and compiler-based systems [33].

In contrast, we explore here a different approach that might appear reactionary in its simplicity but that, in fact, delivers a remarkably sophisticated technology for managing the heterogeneity associated with Grid environments. Specifically, we advocate the use of a well-known low-level parallel programming model, the Message Passing Interface (MPI), as a basis for Grid programming. While not a high-level programming model by any means, MPI incorporates sophisticated support for the management of heterogeneity (e.g., data types), for the construction of modular programs (the communicator construct), for management of latency (asynchronous operations), and for the representation of global operations (collective operations). These and other features have allowed MPI to achieve tremendous success as a standard programming model for parallel computers. We hypothesize that these same features can also be used to good effect for Grid computing.

Our investigation of MPI as a Grid programming model has focused on three related questions. First, can we implement MPI constructs efficiently in Grid environments to hide heterogeneity without introducing overhead? Second, can we use MPI constructs to enable users to manage heterogeneity, when this is required? Third, do users find MPI useful in practice for application development?

To allow for the experimental exploration of these questions, we have developed MPICH-G2, a complete implementation of the MPI-1 standard 42 that uses services provided by the Globus Toolkit ${ }^{T M}$ 17] to extend the popular Argonne MPICH implementation of MPI [27] for Grid execution. MPICH-G2 passes the MPICH test suite and represents a complete redesign and reimplementation of the earlier MPICH-G system [15] that increases performance significantly and incorporates a number of innovations. Our experiences with MPICH-G2, as reported in this article, allow us to respond in the affirmative to each question posed in the preceding paragraph.

MPICH-G2 hides heterogeneity by using Globus Toolkit services for such purposes as authentication, authorization, executable staging, process creation, process monitoring, process control, communication, redirection of standard input and output, and remote file access. The result is that a user can run MPI programs across multiple computers at different sites using the same commands that would be used on a parallel computer. Furthermore, performance studies show that overheads relative to native implementations of basic communication functions are negligible.

MPICH-G2 enables the use of several different MPI features for user management of heterogeneity. MPI's asynchronous operations can be used for latency 
management in wide-area networks. MPI's communicator construct can be used to represent the hierarchical structure of heterogeneous systems and thus allow applications to adapt their behavior to such structures. (In separate work, we present topology-aware collective operations as one example of an "application" [32].) We also show how MPI's communicator construct can be used for user-level management of network quality of service, as first introduced in an earlier article 47.

Many groups have used MPICH-G2 for the execution of both traditional parallel computing applications (e.g., numerical simulation) and nontraditional distributed computing applications (e.g., distributed visualization), in both local-area and widearea networks. This variety of applications and execution environments persuades us that MPI can play a valuable role in Grid computing.

MPICH-G2 is not the only implementation of MPI for heterogeneous systems. Others include MPICH with the ch_p4 device (which provides limited support for heterogeneity), PACX-MPI [23], and STAMPI [36], each of which has interesting features, as we discuss later. Magpie [35], IMPI [31], and PVM [25] also address relevant issues. MPICH-G2 is unique, however, in the degree to which it hides and manages heterogeneity, as well as in its large user community.

In the rest of this article, we describe the problems that we faced in developing MPICH-G2, the techniques used to overcome these problems, and experimental results that indicate the performance of the MPICH-G2 implementation and the extent of its improvement over MPICH-G. We conclude with a discussion of application experiments and future directions.

\section{BACKGROUND}

We first provide some brief background on MPI, MPICH, and the Globus Toolkit.

\subsection{Message Passing Interface}

The Message Passing Interface standard defines a library of routines that implement the message-passing model. These routines include point-to-point communication functions, in which a send operation is used to initiate a data transfer between two concurrently executing program components and a matching receive operation is used to extract that data from system data structures into application memory space; and collective operations such as broadcast and reductions that implement operations involving multiple processes. Numerous other functions address other aspects of message passing, including, in the MPI-2 extensions to MPI [43], single-sided communication and dynamic process creation.

The primary interest of MPI from our perspective, apart from its broad adoption, is the care taken in its design to ensure that underlying performance issues are accessible to, not masked from, the programmer. MPI mechanisms such as asynchronous operations, communicators, and collective operations all turn out to be useful in Grid environments.

\subsection{MPICH Architecture}

MPICH [29] is a popular implementation of the Message Passing Interface standard. It is a high-performance, highly portable library originally developed as a collaborative effort between Argonne National Laboratory and Mississippi State 
University. Argonne continues research and development efforts aimed at improving MPICH performance and functionality.

In its present form, MPICH is a complete implementation of the MPI-1 standard with extensions to support the parallel I/O functionality defined in the MPI-2 standard. It is a mature, widely distributed library, with more than 2,000 downloads per month, not including downloads that occur at mirror sites. Its free distribution and wide portability have contributed materially to the adoption of the MPI standard by the parallel computing community.

MPICH derives its portability from its interfaces and layered architecture. At the top is the MPI interface as defined by the MPI standards. Directly beneath this interface is the MPICH layer, which implements the MPI interface. Much of the code in an MPI implementation is independent of the networking device or process management system. This code, which includes error checking and various manipulations of the opaque objects, is implemented directly at the MPICH layer. All other functionality is passed off to lower layers be means of the Abstract Device Interface (ADI).

The ADI is a simpler interface than MPI proper and focuses on moving data between the MPI layer and the network subsystem. Those interested in implementing MPI for a particular platform need only define the routines in the ADI in order to obtain a full implementation. Existing implementations of this device interface for various MPPs, SMPs, and networks provide complete MPI functionality in a wide variety of environments. MPICH-G2 is another implementation of the ADI and is otherwise known as the globus2 device.

\subsection{The Globus Toolkit}

The Globus Toolkit is a collection of software components designed to support the development of applications for high-performance distributed computing environments, or "Grids" 17, 18]. Core components typically define a protocol for interacting with a remote resource, plus an application program interface (API) used to invoke that protocol. (We introduce the protocols and APIs used within MPICH-G2 below.) Higher-level libraries, services, tools, and applications use core services to implement more complex global functionality. The various Globus Toolkit components are reviewed in [21] and described in detail in online documentation and in technical papers.

\section{MPICH-G2: A GRID-ENABLED MPI}

As noted in the introduction, MPICH-G2 is a complete implementation of the MPI-1 standard that uses Globus Toolkit services to support efficient and transparent execution in heterogeneous Grid environments, while also allowing for application management of heterogeneity. (It also implements client/server management functions found in Section 5.4 of the MPI-2 standard [43]. However, we do not discuss these functions here.)

In this section, we first describe the techniques used to hide heterogeneity during startup and for process management, then the techniques used to effect communication in heterogeneous systems, and finally the support provided for application-level management of heterogeneity. 


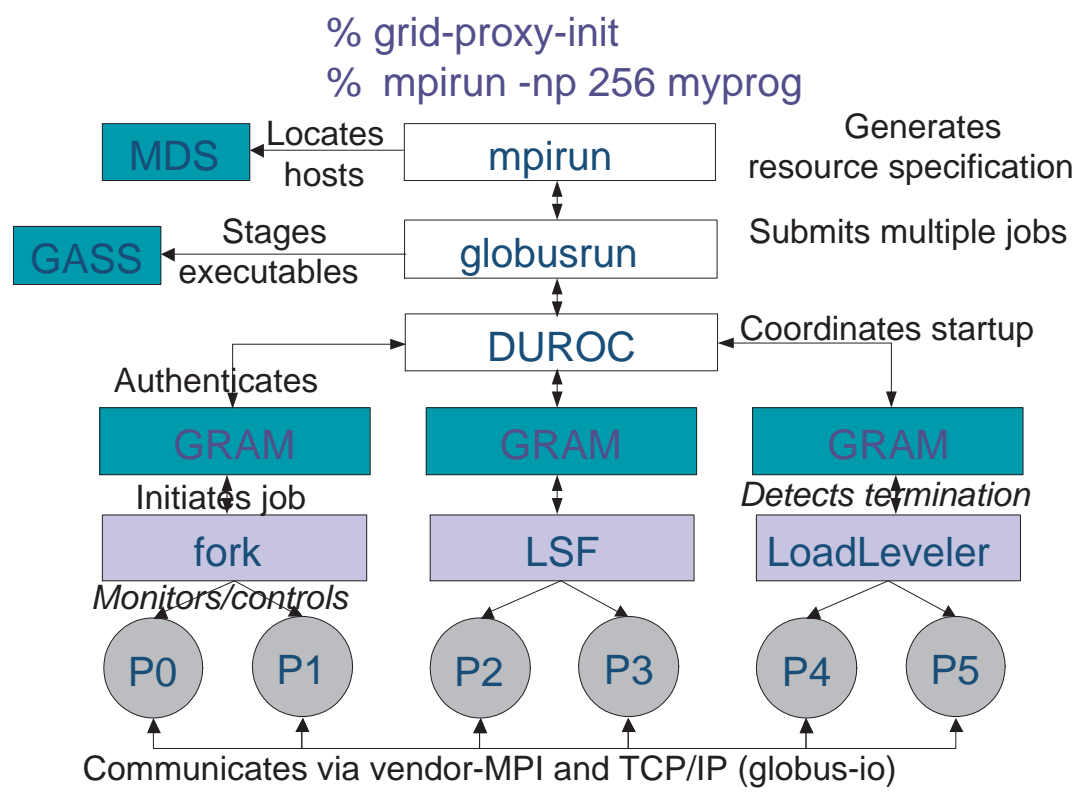

FIG. 1 Schematic of the MPICH-G2 startup, showing the various Globus Toolkit components used to hide and manage heterogeneity. "Fork," "LSF," and "LoadLeveler" are different local schedulers.

\subsection{Hiding Heterogeneity during Startup and Management}

As illustrated in Figure 1 and discussed here, MPICH-G2 uses a range of Globus Toolkit services to address the various complex issues that arise in heterogeneous, multisite Grid environments, such as cross-site authentication, the need to deal with multiple schedulers with different characteristics, coordinated process creation, heterogeneous communication structures, executable staging, and collation of standard output. In fact, MPICH-G2 serves as an exemplary case study of how Globus Toolkit mechanisms can be used to create a Grid-enabled programming tool, as we now explain.

Prior to startup of an MPICH-G2 application, the user employs the Grid Security Infrastructure (GSI) [19] to obtain a (public key) proxy credential that is used to authenticate the user to each remote sites. This step provides a single sign on capability.

The user may also use the Monitoring and Discovery Service (MDS) 13 to select computers on the basis of, for example, configuration, availability, and network connectivity.

Once authenticated, the user uses the standard mpirun command to request the creation of an MPI computation. The MPICH-G2 implementation of this command uses the Resource Specification Language (RSL) [10 to describe the job. In brief, users write $R S L$ scripts, which identify resources (e.g., computers) and specify requirements (e.g., number of CPUs, memory, execution time, etc.) and parameters (e.g., location of executables, command line arguments, environment variables, etc.) for each. Based on the information found in an RSL script, MPICH-G2 calls a co-allocation library distributed with the Globus Toolkit, the Dynamically-Updated 


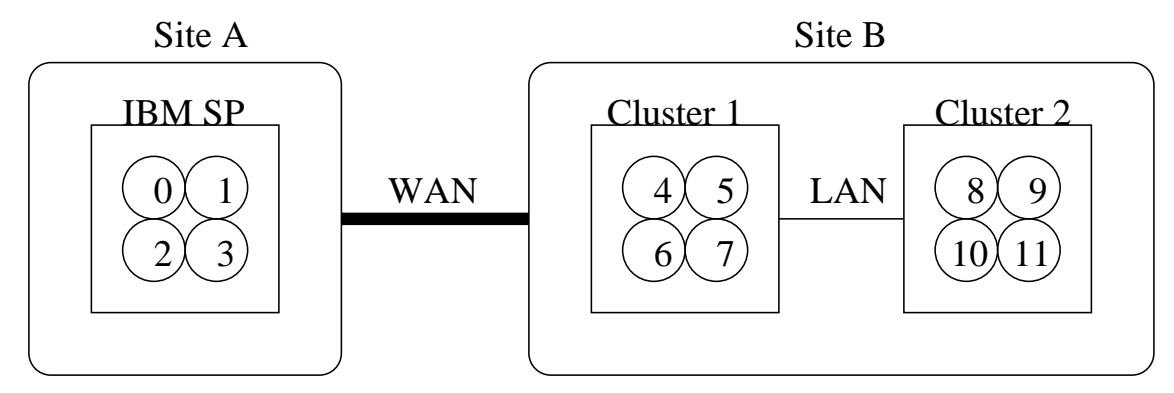

FIG. 2 An example of an MPICH-G2 application running on a computational grid involving 4 processes on an IBM SP at Site A and 8 processes distributed evenly across two Linux clusters at Site B.

Request Online Coallocator (DUROC) 11, to schedule and start the application across the various computers specified by the user.

The DUROC library itself uses the Grid Resource Allocation and Management (GRAM) 10] API and protocol to start and subsequently manage a set of subcomputations, one for each computer. For each subcomputation, DUROC generates a GRAM request to a remote GRAM server, which authenticates the user, performs local authorization, and then interacts with the local scheduler to initiate the computation. DUROC and associated MPICH-G2 libraries tie the various subcomputations together into a single MPI computation.

GRAM will, if directed, use Global Access to Secondary Storage (GASS) [5] to stage executable(s) from remote locations (indicated by URLs). GASS is also used, once an application has started, to direct standard output and error (stdout and stderr) streams to the user's terminal, and to provide access to files regardless of location, thus masking essentially all aspects of geographical distribution except those associated with performance.

Once the application has started, MPICH-G2 selects the most efficient communication method possible between any two processes, using vendor-supplied MPI (vMPI) if available, or Globus communication (Globus IO) with Globus Data Conversion (Globus DC) for TCP, otherwise.

DUROC and GRAM also interact to monitor and manage the execution of the application. Each GRAM server monitors the life cycle of its subcomputation as it passes from pending to running and then to terminating, communicating each state transition back to DUROC. Each subcomputation is held at a DUROCcontrolled barrier and is released from that barrier only after all subcomputations have started executing. Also, a request to terminate the computation ("control C") may be initiated by the user at which time DUROC and the GRAM servers, communicating via GRAM process control messages, terminate all processes.

After the processes have started, MPICH-G2 uses information specified in the RSL script to create multilevel clustering of the processes based on the underlying network topology. Figure 2 depicts an MPI application involving 12 processes distributed across three machines located at two sites. We depict 4 processes (MPI_COMM_WORLD ranks 0-3) on the IBM SP at Site A and 4 processes on each of two Linux clusters (MPI_COMM_WORLD ranks 4-7 and 8-11, respectively) at Site B. Each process in MPI_COMM_WORLD is assigned a topology depth. Processes that communicate using only TCP are assigned topology depths of 3 (to distinguish between 


\begin{tabular}{|c|c|c|c|c|c|c|c|c|c|c|c|c|c|}
\hline & Rank & 0 & 1 & 2 & 3 & 4 & 5 & 6 & 7 & 8 & 9 & 10 & 11 \\
\hline & $\overline{\text { Depth }}$ & 4 & 4 & 4 & 4 & 3 & 3 & 3 & 3 & 3 & 3 & 3 & 3 \\
\hline \multirow{4}{*}{ Colors } & wide area & $\overline{0}$ & $\overline{0}$ & $\overline{0}$ & $\overline{0}$ & $\overline{0}$ & $\overline{0}$ & $\overline{0}$ & $\overline{0}$ & $\overline{0}$ & $\overline{0}$ & $\overline{0}$ & 0 \\
\hline & local area & 0 & 0 & 0 & 0 & 1 & 1 & 1 & 1 & 1 & 1 & 1 & 1 \\
\hline & system area & 0 & 0 & 0 & 0 & 1 & 1 & 1 & 1 & 2 & 2 & 2 & 2 \\
\hline & $v \mathrm{MPI}$ & 0 & 0 & 0 & 0 & & & & & & & & \\
\hline
\end{tabular}

FIG. 3 An example of depths and colors used by MPICH-G2 to represent network topology in a computational grid.

wide area, local area, and intramachine TCP messaging), and processes that can also communicate using a $v$ MPI have a topology depth of 4 . Using these topology depths MPICH-G2 groups processes at a particular level through the assignment of colors. Two processes are assigned the same color at a particular level if they can communicate with each other at the network level.

Figure 3 depicts the topology depths and colors for the processes depicted in Figure 2. Those processes capable of communicating over $v \mathrm{MPI}$, (i.e., those executing on the IBM SP), have a depth of 4 , while the other processes, (i.e., those executing on a Linux cluster), have a depth of 3 . Since all processes are on the same wide-area network, they all have the same color $(0)$ at the wide-area level. Similarly, at the local-area level, all the processes at Site A are assigned one color (0), while all the processes at Site B are assigned another (1). This structure continues through the system-area level, where processes are assigned the same color if and only if they are on the same machine. Finally, processes that can communicate over a $v$ MPI are assigned the same color at the $v \mathrm{MPI}$ level if and only if they can communicate directly with each other over the $v$ MPI.

Topology depths and colors are used in the multilevel topology-aware collective operations and topology-discovery mechanism described in Sections 3.2 and 3.3 , respectively.

\subsection{Heterogeneous Communications}

MPICH-G2 achieves major performance improvements relative to the earlier MPICH-G [15] by replacing Nexus [20], the multimethod, single-sided communication library used for all communication in MPICH-G, with specialized MPICHspecific communication code. While Nexus has attractive features (e.g., multiprotocol support with highly tuned TCP support and automatic data conversion), other attributes have proved less attractive from a performance perspective. MPICH-G2 now handles all communication directly by reimplementing the good things about Nexus and improving the others. The result, as we show in Section 14, is that we achieve performance virtually identical to vendor MPI and MPICH configured with the default TCP (ch_p4) device. We provide here a detailed description of the improvements and additions to MPICH-G used to achieve this impressive performance.

Increased bandwidth. In MPICH-G, each communication involved the copying of data to and from Nexus buffers in sending and receiving processes. MPICH-G2 eliminates these two extra copies in the case of intramachine messages where a 
vendor MPI exists. In this situation, sends and receives now flow directly from and to application buffers, respectively. In addition, for TCP messaging involving basic MPI datatypes (e.g., MPI_INT, MPI_FLOAT) the sending process also transmits directly from the application buffer.

Reduced latency for intramachine vendor MPI messaging. Multiprotocol support is achieved in Nexus by polling each protocol (TCP, vendor MPI, etc.) for incoming messages in a roundrobin fashion [16]. However, this strategy is inefficient in many situations: it is relatively expensive to poll a TCP socket and in practice it is often the case that many processes in a MPICH-G2 computation use only vendor MPI (for communicating with other processes on the same machine).

While this inefficiency can be reduced by adaptive polling [16] or by introducing distinct proxy processes [23, 36], MPICH-G2 takes a more direct approach, exploiting the knowledge about message source that is provided by TCP receive commands to eliminate TCP polling altogether in many situations. MPICH-G2 polls TCP only when the application is expecting data from a source that dictates, or might dictate (e.g., MPI_Recv specifies source=MPI_ANY_SOURCE), TCP messaging.

This avoidance of unnecessary polling when coupled with the need to guarantee progress on both the vendor MPI and TCP protocols leads to implementation decisions that can affect an application's point-to-point communication performance. Specifically, for processes executing on machines where a vendor MPI is available, the context in which the application calls MPI_Recv affects the manner in which MPICH-G2 implements that function, as follows:

- Specified. The source rank specified in the call to MPI_Recv explicitly identifies a process on the same machine (in the same vendor MPI job). Furthermore, no asynchronous requests are outstanding (e.g., incomplete MPI_Irecv and/or MPI_Isend). If these two conditions are met, MPICH-G2 implements MPI_Recv by directly calling the MPI_Recv of the underlying vendor MPI. This is the most favorable circumstances under which an MPI_Recv can be performed.

- Specified-pending. This category is similar to the specified category in that the MPI_Recv specifies an explicit source rank on the same machine. This time, however, one or more unsatisfied receive requests are present, and each such request specifies a source on the same machine. This situation forces MPICH-G2 to continuously poll (MPI_Iprobe) the vendor MPI for incoming messages. This scenario results in less efficient MPICH-G2 performance since the induced polling loop increases latency.

- Multimethod. Here the source rank for the MPI_Recv is MPI_ANY_SOURCE or MPI_Recv is called in the presence of unsatisfied asynchronous requests that require, or might require, TCP messaging. In this situation, MPICH-G2 must poll both TCP and the vendor MPI continuously. This is the least efficient MPICH-G2 scenario, since the relatively large cost of TCP polling results in even greater latency.

In Section 1 , we present a quantitative analysis of the performance differences that result from these different structures. 
More efficient use of sockets. The Nexus single-sided communication paradigm results in MPICH-G2 opening two pairs of sockets between communicating processes and using each pair as a simplex channel (i.e., data always flowing in one direction over each socket pair). MPICH-G2 opens a single pair of sockets between two processes and sends data in both directions. This approach reduces the use of system resources; moreover, by using sockets in the bidirectional manner in which they were intended, it also improves TCP efficiency.

Multilevel topology-aware collective operations. Early implementations of MPI's collective operations sought to construct communication structures that were optimal under the assumption that all processes were equidistant from one another 四, 9. Since this assumption is unlikely to be valid in Grid environments, however, it is desirable that a Grid-enabled MPI incorporate collective operation implementations that take into account the actual topology. MPICH-G2 does this, and we have demonstrated substantial performance improvements for our multilevel topology-aware approach [32] relative both to topology-unaware binomial trees and earlier topology-aware approaches that distinguish only between "intracluster" and "intercluster" communications [30, 35.

As we explain in the next subsection, MPICH-G2's topology-aware collective operations are constructed in terms of topology discovery mechanisms that can also be used by topology-aware applications.

\subsection{Application-Level Management of Heterogeneity}

We have experimented within MPICH-G2 with a variety of mechanisms for application-level management of heterogeneity in the underlying platform. We mention two here.

Topology discovery. Once an MPI program starts, all processes can be viewed as equivalent, distinguished only by their rank. This level of abstraction is desirable from a programming viewpoint but makes it difficult to write programs that exploit aspects of the underlying physical topology, for example, to minimize expensive intercluster communications.

MPICH-G2 addresses this issue within the standard MPI framework by using the MPI communicator construct to deliver topology information to an application. It associates attributes with each MPI communicator to communicate this topology information, which is expressed within each process in terms of topology depths and colors, as described in Section 3.1.

MPICH-G2 applications can then query communicators to retrieve attribute values and structure themselves appropriately. For example, it is straightforward to create new communicators that reflect the underlying network topology. Figure depicts an MPICH-G2 application that first queries the MPICH-G2-defined communicator attributes MPICHX_TOPOLOGY_DEPTHS and MPICHX_TOPOLOGY_COLORS to discover topology depths and colors, respectively, and then uses those values to create three communicators: LANcomm, which groups processes based on site boundaries, VcommA, which groups processes based on their ability to communicate with each other over $v$ MPI, while placing all processes that cannot communicate over $v \mathrm{MPI}$ into a separate communicator, and $\mathrm{VcommB}$, which groups the processes in much the same way as VcommA, but this time does not place processes that cannot 


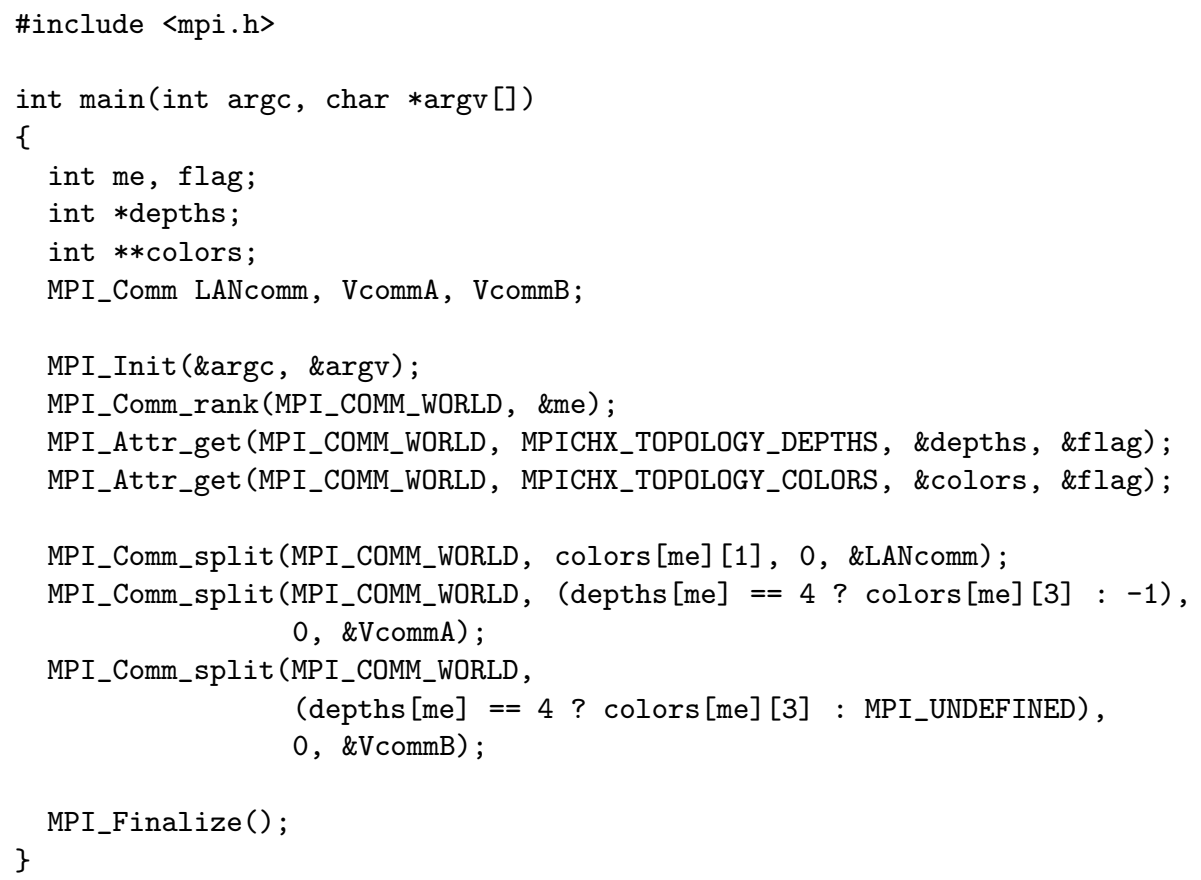

FIG. 4 An example MPICH-G2 application that uses topology depths and colors to create communicators that group processes into various topology-aware clusters.

communicate over $v$ MPI in a communicator (i.e., VcommB is set to MPI_COMM_NULL for those processes).

Quality-of-service management. We have experimented with similar techniques for purposes of quality of service management [47. When running over a shared network, an MPI application may wish to negotiate with an external resource management system to obtain dedicated access to (part of) the network. We show that communicator attributes can be used to set and initiate quality-of-service parameters between selected processes.

\section{PERFORMANCE EXPERIMENTS}

We present the results of detailed performance experiments that characterize the performance of MPICH-G2 and demonstrate the major improvements achieved relative to its predecessor, MPICH-G. We begin by looking at the performance of intramachine communication over a vendor MPI. Then, we examine performance when TCP is the only choice for communicating between a pair of processes. In all cases, mpptest 28, the performance tool included in the MPICH distribution, is used to obtain all results.

\subsection{Vendor MPI}

Evaluating the performance of MPICH-G2 when using a vendor MPI as an underlying communication mechanism is not as simple as running a single set of 


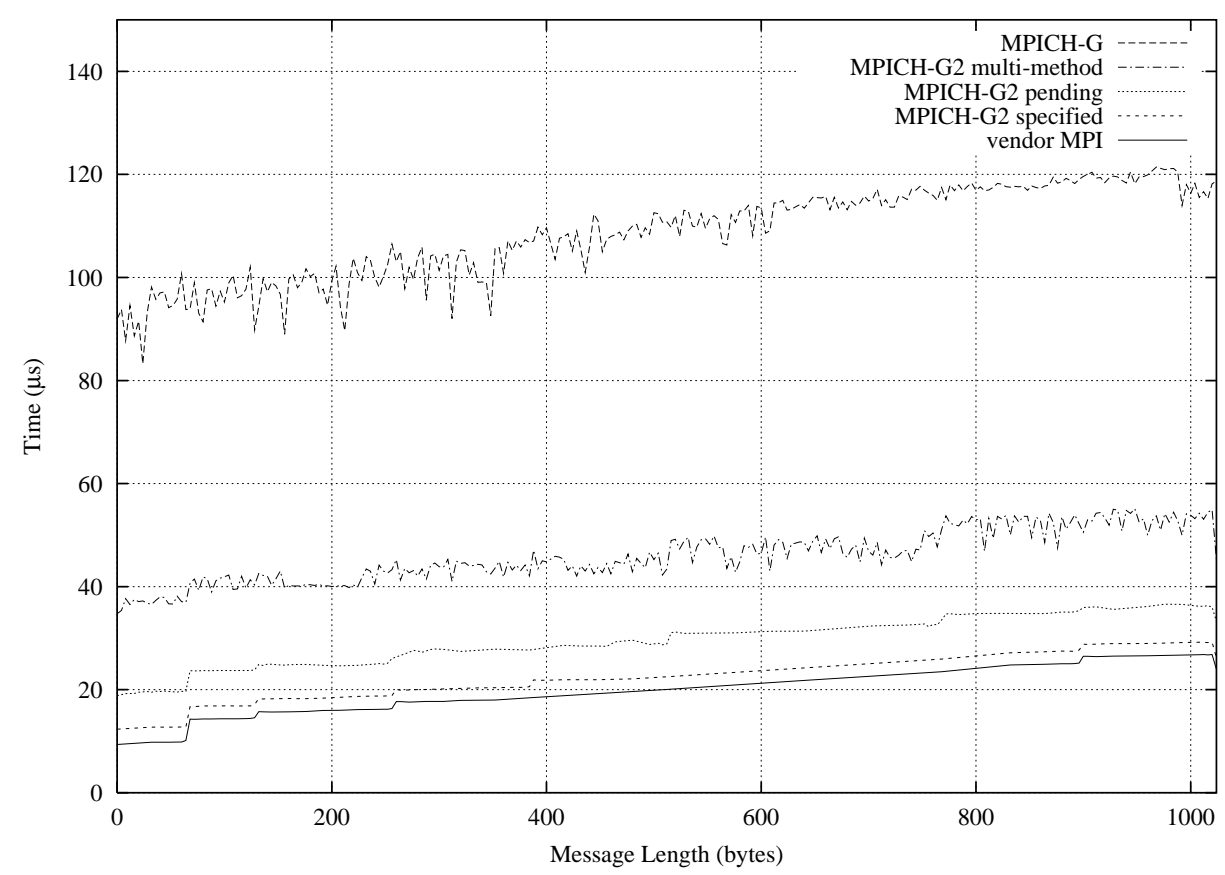

FIG. 5 vMPI experiments - small message latency.

ping-pong tests. As discussed earlier, the performance achieved by MPICH-G2 can be affected by outstanding requests and by the use of MPI_ANY_SOURCE. Therefore, we have divided the experiments into the three categories described in Section 3.2.

Our vendor MPI experiments were run on an SGI Origin2000 at Argonne National Laboratory. Both MPICH-G2 and MPICH-G were built using a nonthreaded, no-debug flavor of Globus 1.1.4 and performed intramachine communication via SGI's implementation of MPI.

One MPICH-G2 design goal was to minimize latency overhead for intramachine communication relative to an underlying vendor MPI. As can been seen in Figure MPICH-G2 does an outstanding job in this regard: only a few extra microseconds of latency are introduced by MPICH-G2 when the source of the message is specified and no other requests are outstanding. In contrast, MPICH-G added approximately 80 microseconds of latency to each message, because the multiple steps required to implement the Nexus single-sided communication model.

The introduction of pending receive requests has a modest impact on MPICH-G2 message latencies. Messages falling into the specified-pending category incur slightly more overhead, as the MPICH-G2 progress engine must continuously poll (probe) the vendor MPI rather than blocking in a receive. Overall, MPICH-G2 latencies increase by several microseconds relative to the first case but are still far less than those of MPICH-G.

The use of MPI_ANY_SOURCE has the largest impact on MPICH-G2 performance. The additional cost is associated with having to poll TCP as well as the vendor MPI. Polling TCP increases the latency of messages by nearly 20 microseconds over those in the specified-pending category. While the increase is significant, however, 


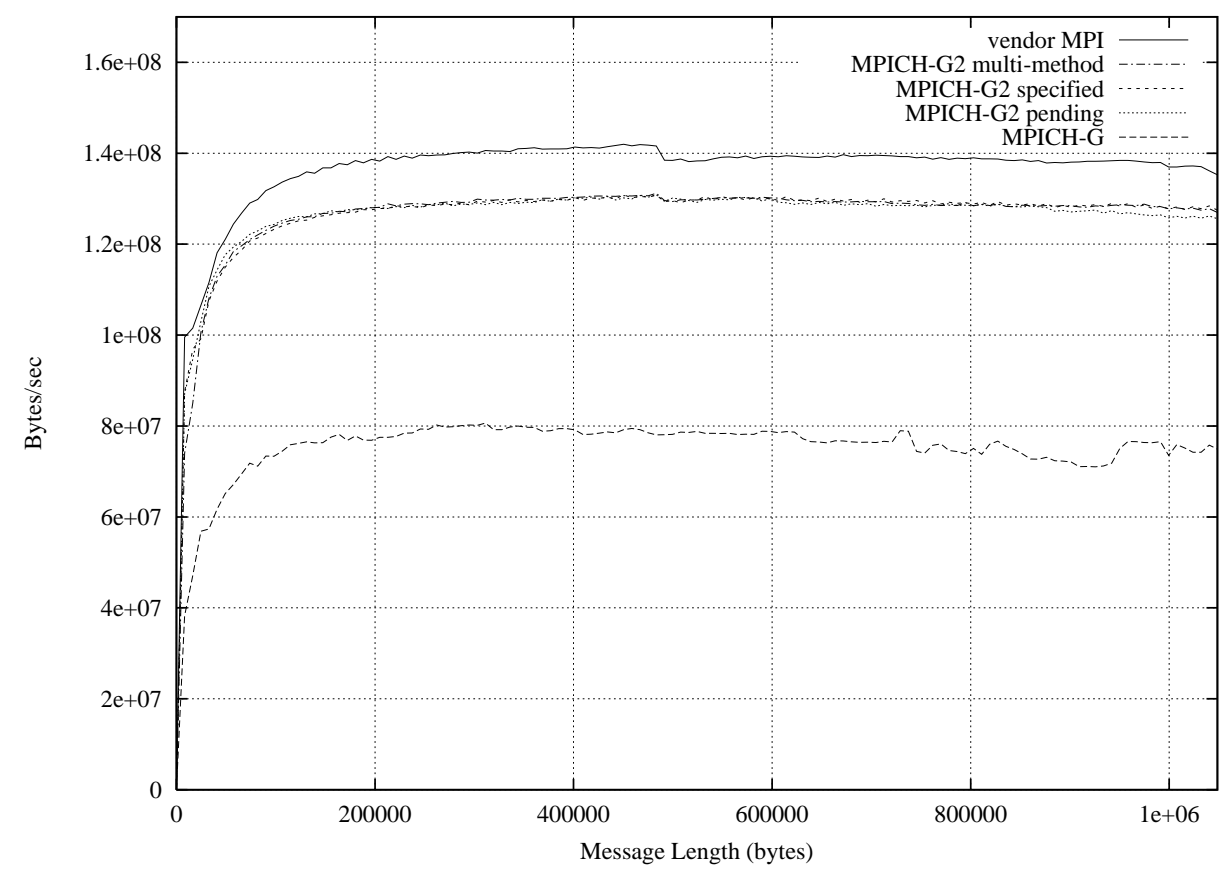

FIG. 6 vMPI experiments - realized bandwidth.

these latencies are still considerably less than for MPICH-G.

While MPICH-G2 message latencies are affected by the use of MPI_ANY_SOURCE and pending receive requests, the realized bandwidths are largely unaffected. Figure 6 shows the bandwidths obtained for messages up to one megabyte. We see that the bandwidths for MPICH-G2 are nearly identical for all but small messages. While the large message bandwidths for MPICH-G2 are approximately $7 \%$ less than those for the the vendor MPI (for reasons we do not yet understand), they represent an improvement of more than $60 \%$ over MPICH-G.

\section{2. $\mathrm{TCP} / \mathrm{IP}$}

Performance optimization work on MPICH-G2 performed to date has focused on intramachine messaging when a vendor MPI is used as the underlying communication mechanism. The MPICH-G2 TCP/IP communication code has not been optimized. However, its performance is quite reasonable when compared with MPICH-G and to MPICH configured with the default TCP (ch_p4) device.

All TCP/IP performance measurements were taken using a pair of SUN workstations in Argonne's Mathematics and Computer Science Division. These two machines were connected to a local-area network via gigabit Ethernet. Both MPICH-G and MPICH-G2 were built using a nonthreaded, no-debug flavor of Globus 1.1.4.

Figure 7 shows the small message latencies exhibited by all three systems. We see that for most message sizes, MPICH-G2 is $20 \%$ to $30 \%$ slower than MPICH/ch_p4, although the difference is much smaller for very small messages. We also see that MPICH-G2 latencies, in most cases, are somewhat less than those of MPICH-G. 


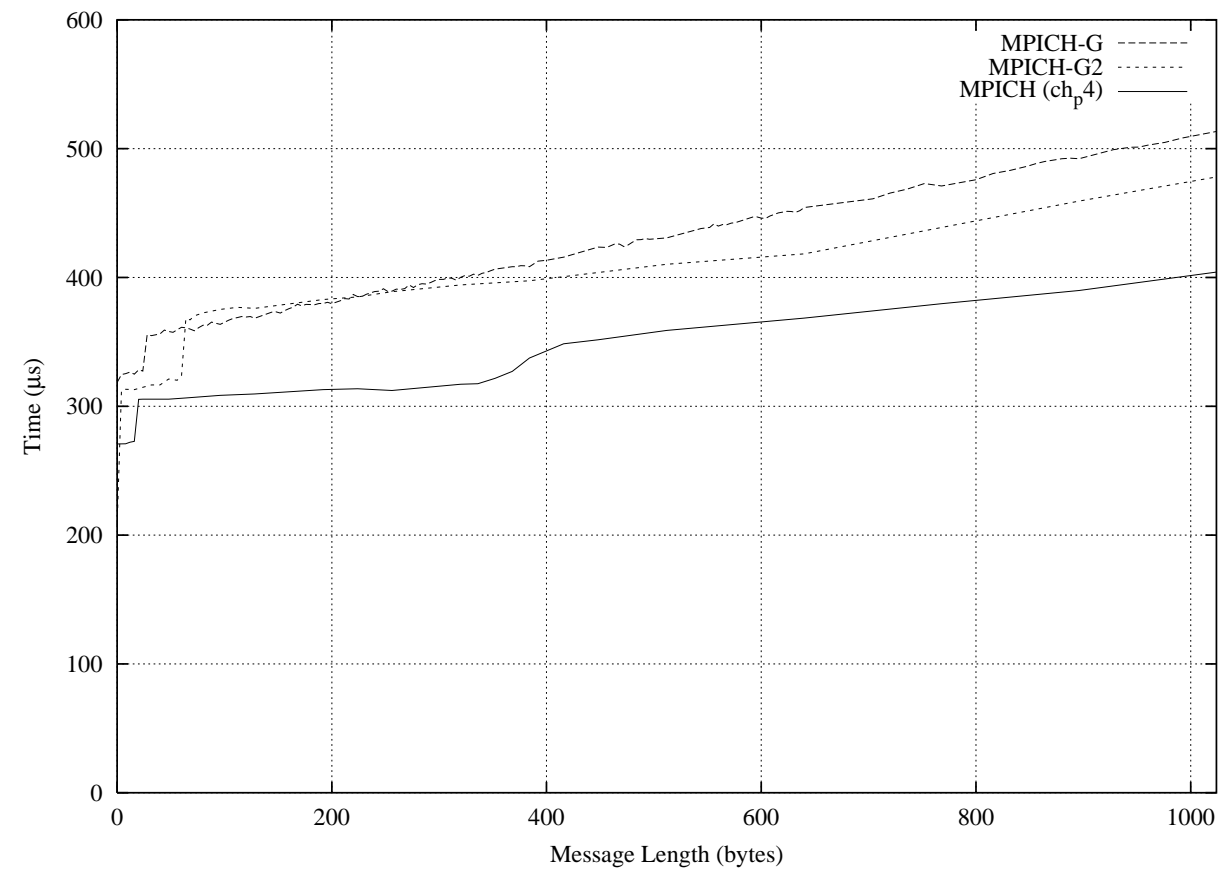

FIG. 7 TCP/IP experiments - small message latency.

The most notable data point is barely visible on the graph but emphasizes a clear optimization that is missing in MPICH-G2. The latency for zero-byte messages is 140 microseconds, while the latency for an eight-byte message is $224 \mathrm{mi}$ croseconds. The reason for this large difference is that MPICH-G2 currently uses separate system calls to send the message header and the message data. This data point suggests that by combining these two writes into a single vector write, we could reduce the latency of small messages significantly. While this difference might seem unimportant for machines separated by a wide-area network, it can be significant when MPICH-G2 is used to combine multiple machines with the same machine room or even at the same site.

Figure 8 shows the bandwidths obtained by all three systems for message sizes up to one megabyte. For large messages, we see that MPICH-G2 performs approximately $5 \%$ better than the other two systems. This improvement is a result of the message data being sent directly from the user buffer rather than being copied into a separate buffer before write is called. For preposted receives with contiguous data, further improvement is possible. Data for these receives can be read directly into the user buffer, avoiding a buffer copy that, at present, always takes place at the receiver.

\section{APPLICATION EXPERIENCES}

MPICH-G2 has been used by many groups worldwide for a wide variety of purposes. Here we mention a few relevant experiences that highlight interesting features of the system. 


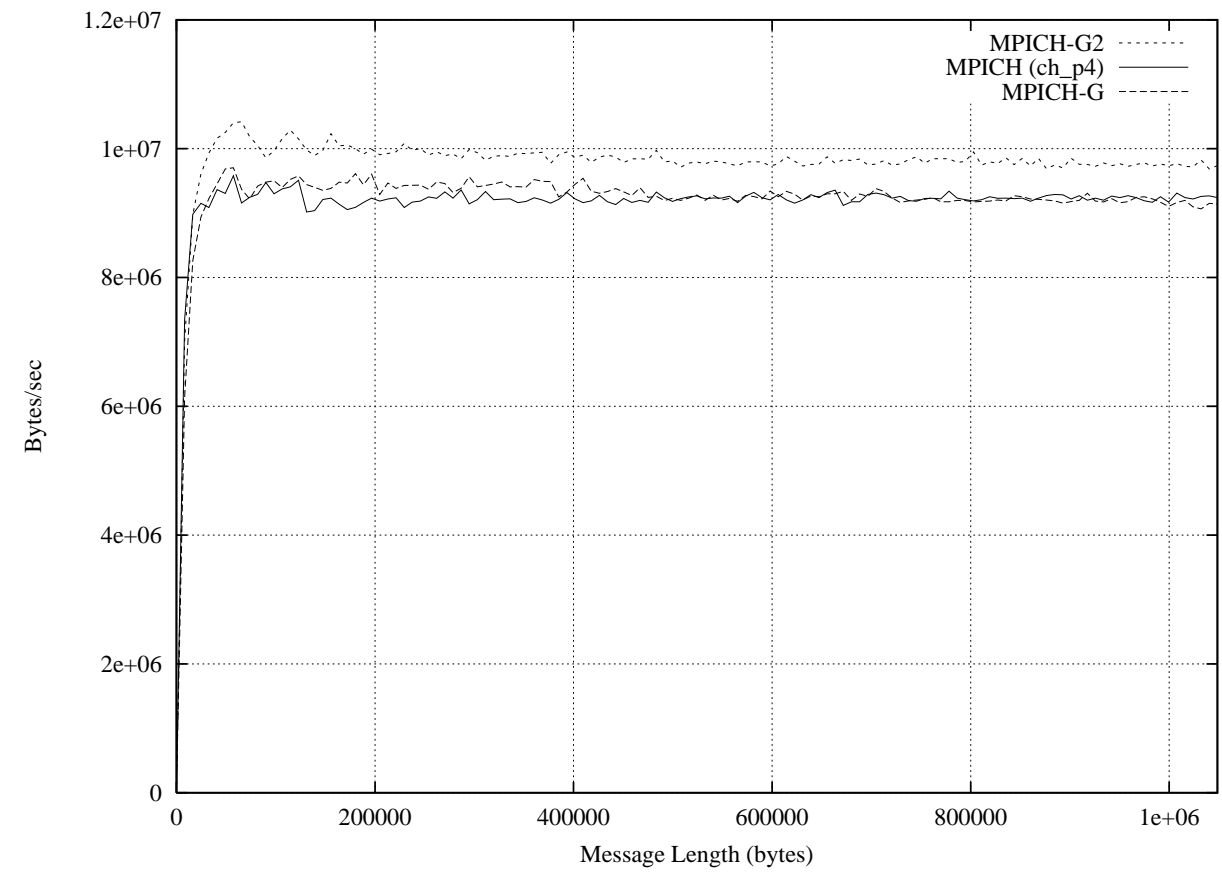

FIG. 8 TCP/IP experiments - realized bandwidth.

One interesting use of MPICH-G2 is to run conventional MPI programs across multiple parallel computers within the same machine room. In this case, MPICH-G2 is used primarily to manage startup and to achieve efficient communication via use of different low-level communication methods. Other groups are using MPICH-G2 to distribute applications across computers located at different sites, for example, Taylor performing MM5 climate modeling on the NSF TeraGrid [49, 46], Mahinthakumar forming multivariate geographic clusters to produce maps of regions of ecological similarity [41, Larsson for studies of distributed execution of a large computational electromagnetics code [38], and Chen and Taylor in studies of automatic partitioning techniques, as applied to finite element codes 8 .

MPICH-G2 has also been successfully used in demonstrations that promote MPI as an application-level interface to Grids for nontraditional distributed computing applications, for example, Roy et al. for studies in using MPI idioms for setting QoS parameters 477 and Papka and Binns for creating distributed visualization pipelines using MPICH-G2's client/server MPI-2 extensions 49, 46.

\section{MPICH-G2}

was awarded a 2001 Gordon Bell Award for its role in an astrophysics application used for solving problems in numerical relativity to study gravitational waves from colliding black holes [2]. The winning team used MPICH-G2 to run across four supercomputers in California and Illinois, achieving scaling of $88 \%$ (1,140 CPUs) and $63 \%(1,500 \mathrm{CPUs})$ computing a problem size five times larger than any other previous run. 


\section{FUTURE WORK}

The successful development of MPICH-G2 and its widespread adoption both make it a useful platform for future research and create significant interest in its continued development.

One immediate area of concern is full support for MPI-2 features. In particular, support for dynamic process management will allow MPICH-G2 to be used for a wider class of Grid computations in which either application requirements or resource availability changes dynamically over time. The necessary support exists in the Globus Toolkit, and so this work depends primarily on the availability of the next-generation ADI-3. Less obvious, but very interesting, is how to integrate support for fault tolerance into MPICH-G2 in a meaningful way.

A second area of concern relates to exploring and refining MPICH-G2 support for application-level management of heterogeneity. Initial experiments with topology discovery and quality-of-service management have been encouraging, but it seems inevitable that application experiences will reveal deficiencies in current techniques or suggest additional MPICH-G2 support that could further improve application flexibility.

Our work on collective operations can be improved in various ways. In particular, van de Geijn et al. 3] have shown that are advantages in implementing collective operations by segmenting and pipelining messages when communicating over relatively slower channels (e.g., TCP over local- and wide-area networks). These pipelining techniques can be used throughout many of the levels in MPICH-G2's multilevel topology-aware collective operations.

\section{RELATED WORK}

A variety of approaches have been proposed to programming Grid applications, including object systems [26, 24], Web technologies [22, 50], problem solving environments [7, 15, CORBA, workflow systems, high-throughput computing systems [1, 39, and compiler-based systems [33]. We assume that while different technologies will prove attractive for different purposes, a programming model such as MPI that allows direct control over low-level communications will always be attractive for certain applications.

Other systems that support message passing in heterogeneous environments include the pioneering Parallel Virtual Machine (PVM) [25, 48] and the PACXMPI [23], MetaMPI [12], and STAMPI [36] implementations of MPI, each of which addresses issues relating to efficient communication in heterogeneous wide-area systems. STAMPI supports MPI-2 dynamic process management features. PACXMPI, like MPICH-G2, supports the automatic startup of distributed computations, but uses ssh rather than the GRAM protocol with its integrated GSI authentication, for that purpose; nor does it address issues of executable staging. PACX-MPI (and STAMPI) also differ in how it addresses wide-area communication. While in MPICH-G2, any processor may speak both local and wide-area communication protocols, PACX-MPI and STAMPI forward all off-cluster communication operations to an intermediate gateway node.

Other implementations of MPI include MPICH with the ch_p4 device and LAM/MPI [6, 37]. By contrast these implementations were designed for local area networks and not computational grids. 
The Interoperable MPI (IMPI) standards effort [31] defines standard message formats and protocols with a view to enabling interoperability among different MPI implementations. IMPI does not address issues of computation management and control; in principle, the techniques developed within MPICH-G2 could be used for that purpose.

Other related projects include MagPIe [35] and MPI-StarT [30], which show how careful consideration of communication topologies can result in significant improvements after modifying the MPICH broadcast algorithm, which uses topologyunaware binomial trees. However, both limit their view of the network to only two layers; processors are either near or far. Further performance improvements can be realized by adopting the multilevel network view. We referred in the preceding section to the work of van de Geijn et al. 33. In 34 Kielman et al. have extended MagPIe by incorporating van de Geijn's pipelining idea through a technique they call Parameterized $\log \mathrm{P}(\mathrm{P} \log \mathrm{P})$, which is an extension of the $\log \mathrm{P}$ model presented by Culler et al [9]. In this extension, MagPIe still recognizes only a two-layer communication network, but through parameterized studies of the network they determine "optimal" packet sizes.

Various projects have investigated programming model extensions to enable application management of QoS, for example, Quo 40]. The only other relevant effort in the context of MPI is work on real-time extensions to MPI. MPI/RT [44] provides a QoS interface but is not an established standard and introduces a new programming interface. Furthermore, the focus is on real-time needs such as predictability of performance and system resource usage more appropriate for embedded systems than for wide-area networks.

\section{SUMMARY}

We have described MPICH-G2, an implementation of the Message Passing Interface that uses Globus Toolkit mechanisms to support the execution of MPI programs in heterogeneous wide-area environments. MPICH-G2 masks details of underlying networks, software systems, policies, and computer architectures so that diverse distributed resources can appear as a single MPI_COMM_WORLD. Arbitrary MPI applications can be started on heterogeneous collections of machines simply by typing mpirun: authentication, authorization, executable staging, resource allocation, job creation, startup, and routing of stdout and stderr are all handled automatically via Globus Toolkit mechanisms. MPICH-G2 also enables the use of MPI features for user-level management of heterogeneity, for example, via the use of MPI's communicator construct to access system topology information. A wide range of successful application experiences have demonstrated MPICH-G2's utility in practical settings, both for traditional simulation applications and for less traditional applications such as distributed visualization pipelines.

While MPICH-G2 is already a sophisticated tool that is seeing widespread use, there are also several areas in which it can be extended and improved. Support for MPI-2 features, in particular dynamic process management, will be invaluable for Grid applications that adapt their resource usage to changing conditions and application requirements. This support will be provided as soon as it is incorporated into MPICH. More challenging is the design of techniques for effective fault management, a major topic for future research. Here we may be able to draw upon techniques developed within systems such as PVM 25. 


\section{ACKNOWLEDGMENTS}

We thank Olle Larsson and Warren Smith for early discussions and for prototyping the techniques that enable us to use vendor-supplied MPI. MPICH-G2 is, to a large extent, the result of our MPICH-G experiences. We therefore thank Jonathan Geisler, who originally designed and implemented MPICH-G while at Argonne, and George Thiruvathukal, who further developed MPICH-G also while at Argonne. We thank William Gropp, Ewing Lusk, David Ashton, Anthony Chan, Rob Ross, Debbie Swider, and Rajeev Thakur of the MPICH group at Argonne for their guidance, assistance, insight, and many discussions. We thank Sebastien Lacour for his efforts in conducting the performance evaluation and his many other contributions. His insight and ingenuity were invaluable to the implementation of the topology-aware components of MPICH-G2. Finally, we thank all the members of the Globus development team for their support, patience, and many ideas.

This work was supported in part by the Mathematical, Information, and Computational Sciences Division subprogram of the Office of Advanced Scientific Computing Research, U.S. Department of Energy, under Contract W-31-109-Eng-38; by the U.S. Department of Energy under Cooperative Agreement No. DE-FC02-99ER25398; by the Defense Advanced Research Projects Agency under contract N66001-96-C-8523; by the National Science Foundation; and by the NASA Information Power Grid program.

\section{REFERENCES}

[1] D. Abramson, R. Sosic, J. Giddy, and B. Hall. Nimrod: A tool for performing parameterised simulations using distributed workstations. In Proc. 4th IEEE Symp. on High Performance Distributed Computing. IEEE Computer Society Press, 1995.

[2] G. Allen, T. Dramlitsch, I. Foster, M. Ripeanu N. T. Karonis, E. Seidel, and B. Toonen. Supporting efficient execution in heterogeneous distributed computing environments with catus and globus. In Proceedings of Supercomputing 2001. IEEE Computer Society Press, 2001, winner Gordon Bell Award, Special Category.

[3] M. Barnett, R. Littlefield, D. Payne, and R. van de Geijn. On the efficiency of global combine algorithms for 2-d meshes with wormhole routing. Journal of Parallel and Distributed Computing, 22:324-328, 1994.

[4] A. Bary-Noy and S. Kipnis. Designing broadcasting algorithms in the postal model for message-passing systems. In Proceedings of the 4th Annual ACM Symposium on Parallel Algorithms and Architectures, pages 559-566, June 1992.

[5] Joseph Bester, Ian Foster, Carl Kesselman, Jean Tedesco, and Steven Tuecke. GASS: A data movement and access service for wide area computing systems. In Proc. IOPADS'99. ACM Press, 1999.

[6] Greg Burns, Raja Daoud, and James Vaigl. LAM: An open cluster environment for MPI. In John W. Ross, editor, Proceedings of Supercomputing Symposium '94, pages 379-386. University of Toronto, 1994.

[7] Henri Casanova and Jack Dongarra. Netsolve: A network server for solving computational science problems. Technical Report CS-95-313, University of Tennessee, November 1995. 
[8] Jian Chen and Valerie Taylor. Mesh partitioning for distributed systems. In Proc. 7th IEEE Symp. on High Performance Distributed Computing. IEEE Computer Society Press, 1998.

[9] D.E. Culler, R. Karp, D.A. Patterson, A. Sahay. K.E. Schauser, E. Santos, R. Subramonian, and T. von Eicken. Logp: Towards a realistic model of parallel compuation. In Proceedings of the 4th SIGPLAN Symposium on Principles and Practices of Parallel Programming, pages 1-12, May 1993.

[10] K. Czajkowski, I. Foster, N. Karonis, C. Kesselman, S. Martin, W. Smith, and S. Tuecke. A resource management architecture for metacomputing systems. In The 4th Workshop on Job Scheduling Strategies for Parallel Processing, 1998.

[11] Karl Czajkowski, Ian Foster, and Carl Kesselman. Co-allocation services for computational grids. In Proc. 8th IEEE Symp. on High Performance Distributed Computing. IEEE Computer Society Press, 1999.

[12] Thomas Eickermann, Helmut Grund, and Jorg Henrichs. Performance issues of distributed mpi applications in a german gigabit testbed. In Proceedings of the 6th European PVM/MPI Users' Group Meeting, September 1999.

[13] S. Fitzgerald, I. Foster, C. Kesselman, G. von Laszewski, W. Smith, and S. Tuecke. A directory service for configuring high-performance distributed computations. In Proc. 6th IEEE Symp. on High Performance Distributed Computing, pages 365-375. IEEE Computer Society Press, 1997.

[14] I. Foster. The grid: A new infrastructure for 21st century science. Physics Today, 54(2), 2002.

[15] I. Foster, J. Geisler, W. Gropp, N. Karonis, E. Lusk, G. Thiruvathukal, and S. Tuecke. A wide-area implementation of the Message Passing Interface. Parallel Computing, 24(12):1735-1749, 1998.

[16] I. Foster, J. Geisler, C. Kesselman, and S. Tuecke. Managing multiple communication methods in high-performance networked computing systems. Journal of Parallel and Distributed Computing, 40:35-48, 1997.

[17] I. Foster and C. Kesselman. The Globus project: A status report. In Proceedings of the Heterogeneous Computing Workshop, pages 4-18. IEEE Computer Society Press, 1998.

[18] I. Foster and C. Kesselman, editors. The Grid: Blueprint for a New Computing Infrastructure. Morgan Kaufmann Publishers, 1999.

[19] I. Foster, C. Kesselman, G. Tsudik, and S. Tuecke. A security architecture for computational grids. Technical report, Mathematics and Computer Science Division, Argonne National Laboratory, Argonne, Ill., 1998.

[20] I. Foster, C. Kesselman, and S. Tuecke. The Nexus approach to integrating multithreading and communication. Journal of Parallel and Distributed Computing, 37:70-82, 1996.

[21] I. Foster, C. Kesselman, and S. Tuecke. The anatomy of the grid: Enabling scalable virtual organizations. International Journal of High Performance Computing Applications, 15(3):200-222, 2001. 
[22] Geoffrey Fox and Wojtek Furmanski. High-performance commodity computing. In [1] , pages 237-255.

[23] Edgar Gabriel, Michael Resch, Thomas Beisel, and Rainer Keller. Distributed computing in a heterogenous computing environment. In Proc. EuroPVMMPI'98. 1998.

[24] Dennis Gannon and Andrew Grimshaw. Object-based approaches. In [18], pages 205-236.

[25] A. Geist, A. Beguelin, J. Dongarra, W. Jiang, B. Manchek, and V. Sunderam. PVM: Parallel Virtual Machine-A User's Guide and Tutorial for Network Parallel Computing. MIT Press, 1994.

[26] A. S. Grimshaw, W. A. Wulf, and the Legion team. The Legion vision of a worldwide virtual computer. Communications of the ACM, 40(1), January 1997.

[27] W. Gropp, E. Lusk, N. Doss, and A. Skjellum. A high-performance, portable implementation of the MPI message passing interface standard. Parallel Computing, 22:789-828, 1996.

[28] William Gropp and Ewing Lusk. Reproducible measurements of MPI performance characteristics. Technical Report ANL/MCS-P755-0699, Mathematics and Computer Science Division, Argonne National Laboratory, June 1999.

[29] William Gropp, Ewing Lusk, Nathan Doss, and Anthony Skjellum. A highperformance, portable implementation of the MPI Message-Passing Interface standard. Parallel Computing, 22(6):789-828, 1996.

[30] P. Husbands and J.C. Hoe. MPI-StarT: Delivering network performance to numerical applications. In Proceedings of Supercomputing '98, November 1998.

[31] Interoperable mpi web page. http://impi.nist.gov.

[32] N. Karonis, B. de Supinski, I. Foster, W. Gropp, E. Lusk, and J. Bresnahan. Exploiting hierarchy in parallel computer networks to optimize collective operation performance. In Proceedings of the 14th International Parallel and Distributed Processing Symposium, 2000.

[33] Ken Kennedy. Compilers, languages, and libraries. In [1]], pages 181-204.

[34] T. Kielmann, H. E. Bal, S. Gorlatch, K. Verstoep, and R. F. H. Hofman. Network performance-aware collective communication for clustered wide area systems. Parallel Computing, 2001. accepted for publication.

[35] T. Kielmann, R.F.H. Hofman, H.E. Bal, A. Plaat, and R.A.F. Bhoedjang. MAGPIE: MPI's collective communcation operations for clustered wide area systems. In Proceedings of Supercomputing '98, November 1998.

[36] T. Kimura and H. Takemiya. Local area metacomputing for multidisciplinary problems: A case study for fluid/structure coupled simulation. In Proc. Intl. Conf. on Supercomputing, pages 145-156. 1998.

[37] Collected LAM documents. World Wide Web. ftp://tbag. osc.edu/pub/lam. 
[38] Olle Larsson. Implementation and performance analysis of a high-order CEM algorithm in parallel and distributed environments. Master's thesis, University of Houston, 1998.

[39] M. Litzkow, M. Livny, and M. Mutka. Condor - a hunter of idle workstations. In Proc. 8th Intl Conf. on Distributed Computing Systems, pages 104-111, 1988.

[40] J. P. Loyall, R. E. Schantz, J. A. Zinky, and D. E. Bakken. Specifying and measuring quality of service in distributed object systems. In Proceedings of the First International Symposium on Object-Oriented Real-Time Distributed Computing (ISORC '98), 1998. Kyoto, Japan.

[41] G. Mahinthakumar, F. M. Hoffman, W. W. Hargrove, and N. Karonis. Multivariate geographic clustering in a metacomputing environment using globus. In Proceedings of Supercomputing '99. IEEE Computer Society Press, 1999.

[42] Message Passing Interface Forum. MPI: A message-passing interface standard. International Journal of Supercomputer Applications, 8(3/4):165-414, 1994.

[43] Message Passing Interface Forum. MPI2: A message passing interface standard. International Journal of High Performance Computing Applications, 12(1-2):1-299, 1998.

[44] Mpi/rt forum. http://www.mpirt.org.

[45] Hidemoto Nakada, Mitsuhisa Sato, and Satoshi Sekiguchi. Design and implementations of ninf: towards a global computing infrastructure. Future Generation Computing Systems, 15:649-658, 1999.

[46] Ncsa press release web page. http://www.ncsa.edu/News/Access/Releases/011211. TeraGrid.html.

[47] A. Roy, I. Foster, W. Gropp, N. Karonis, V. Sander, and B. Toonen. MPICHGQ: Quality-of-Service for message passing programs. In Proceedings of Supercomputing 2000. IEEE Computer Society Press, 2000.

[48] T. Sheehan, W. Shelton, T. Pratt, P. Papadopoulos, P. LoCascio, and T. Dunigan. Locally self consistent multiple scattering method in a geographically distributed linked MPP environment. Parallel Computing, 24, 1998.

[49] Teragrid web page. http://www.teragrid.org.

[50] Amin Vahdat, Eshwar Belani, Paul Eastham, Chad Yoshikawa, Thomas Anderson, David Culler, and Michael Dahlin. WebOS: Operating system services for wide area applications. In 7th Symposium on High Performance Distributed Computing, July 1998.

Nicholas T. Karonis received a B.S. in finance and a B.S. in computer science from Northern Illinois University in 1985, an M.S. in computer science from Northern Illinois University in 1987, and a Ph.D. in computer science from Syracuse University in 1992. He spent summers from 1981 to 1991 as a student at Argonne National Laboratory, where he worked on the p4 message-passing library, automated reasoning, and genetic sequence alignment. From 1991 to 1995 he worked on the control system at Argonne's Advanced Photon Source and from 1995 to 
1996 for the Computing Division at Fermi National Accelerator Laboratory. Since 1996 he has been an assistant professor of computer science at Northern Illinois University and a resident associate guest of Argonne's Mathematics and Computer Science Division where he has been a member of the Globus Project. His current research interests is message- passing systems in computational grids.

Brian Toonen received his B.S. in computer science from the University of Wisconsin Oshkosh in 1993, and his M.S. in computer science from the University of Wisconsin-Madison in 1997. He is a senior scientific programmer with the Mathematics and Computer Science Division at Argonne National Laboratory. Brian's research interests include parallel and distributed computing, operating systems, and networking. He is currently working with the MPICH team to create a portable, high-performance implementation of the MPI-2 standard. Prior to joining the MPICH team, he was a senior developer for the Globus Project.

Ian Foster received his B.Sc. (Hons I) at the University of Canterbury in 1979 and his Ph.D. from Imperial College, London, in 1998. He is a senior scientist and associate director of the Mathematics and Computer Science Division at Argonne National Laboratory, and professor of computer science at the University of Chicago. He has published four books and over 150 papers and technical reports. He co-leads the Globus Project, which provides protocols and services used by industrial and academic distributed computing projects worldwide. He co-founded the influential Global Grid Forum and co-edited the book "The Grid: Blueprint for a New Computing Infrastructure." 